(Aus dem physiologischen Institut der Universität Rostock.)

\title{
Vergleichende \\ Versuche über die Wirkung rhythmischer und kontinuierlicher Durchspülung.
}

Von

Paul Gerlach.

(Mit 1 Textfigur.)

Fast alle Physiologen, die einen Apparat konstruiert haben, um ganze Tiere oder rur einzelne "überlebende" Organe mit Blut oder einer blutersetzenden Flüssigkeit zu versorgen, haben besonderen Wert darauf gelegt, die Durchspülung den natürlichen Zirkulationsverhältnissen möglichst ähnlich zu gestalten. Sie waren also vor allem bestrebt, den Rhythmus, den die Natur durch den Herzschlag hervorbringt, bei ihren Versuchen irgendwie künstlich nachzuabmen. Hören wir über diesen Punkt die Autoren selbst.

Kronecker ${ }^{1}$ sagt: "Viel besser vertragen die Gefässe der Froschmuskeln ganz kurz dauernde periodische Druckerhöhungen" (als eine kontinuierliche Durchspülung). Ferner heisst es in Brodies Abhandlung "The perfusion of surviving Organs" ${ }^{2}$ ): „In many cases it is advisable to vary the pressure of the blood-supply rythmically in imitation of the pulsation of an artery". Dementsprechend hat Brodie dann auch einen Durchspülungsapparat ${ }^{3}$ ) konstruiert, bei dem eine Pumpe ein stossweises Durchfliessen der Flüssigkeit durch das Gefässsystem des Tieres bewirkte. Ebenso verwendeten

1) H. Kronecker, Über die Ermüdung und Erholung der quergestreiften Muskeln. Ber. üb. d. Verhandl. d. Kgl. sächs. Gesellsch. d. Wissensch. zu Leipzig, math.-phys. Klasse 1871.

2) Brodie, Journ. of Physiol. vol. 29 p. 269. 1903.

3) Du Bois-Reymond, Brodie, F. Müller, Viskosität und Blutströmung. Arch. f. Anat. u. Physiol. 1907 Suppl. S. 41. 
noch Frey und Gruber ${ }^{1}$ ), J. Ries ${ }^{2}$ ) und eine Reihe anderer Forscher, die $\mathrm{Skutul}^{3}$ ) in einer Arbeit „Über Durchströmungs-

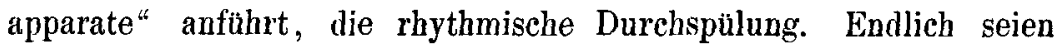
hierzu noch die Ansichten einiger Autoren aus neuester Zeit erwähnt. Winterstein ${ }^{4}$ ) äussert sich in einer Abhandlung "Zur Kenntnis der Narkose" folgendermassen: „Es hat sich indessen als zweckmässig herausgestellt, an die Stelle der kontinuierlichen Durchströmung eine den natürlichen Verhältnissen besser entsprechende, rhythmische, stossweise Durchspülung zu setzen, was am einfachsten durch rhy thmische Kompression des Spülschlauches erzielt werden kann". Ganz ähnlich drückt sich Amedeo Herlitzka ${ }^{5}$ ) aus: $„ \ldots$ und der D́ruck, der das Blut beförderte, war ein rhythmischer und kein beständiger, was ja bekanntlich bei allen Durchblutungsversuchen von grosser Wichtigkeit ist". Auch Verworn ${ }^{6}$ ) vertritt dieselbe Ansicht: „Es hat sich nämlich gezeigt, dass rhythmische Schwankungen des Flüssigkeitsdruckes im allgemeinen bessere Resultate geben als eine kontinuierliche Durchspülung der Gefässe unter konstantem Druck, offenbar weil bei konstantem Druck häufig einzelne kleinste Kapillargefässe, die momentan undurchgängig sind, dauernd undurchgängig bleiben, während sie infolge der rhythmischen Druckschwankungen wieder durchgängig werden."

Sehen wir nun zu, welche Vorteile der rhythmischen Durchspülung gegenüber der kontinuierlichen die Autoren zur Bevorzugung der ersteren bei ihren Durchblutungsversuchen bewogen haben.

Max von Frey ${ }^{7}$ ) stellte bei konstantem Druck „eine eigentümliche Verstopfung der Kapillaren mit Blutkörperchen" fest, wodurch der Widerstand in dem Gefässsystem während des Versuches wuchs. Diese Erscheinung blieb fast ganz aus, wenn der Druck stossweise

1) Frey und Gruber, Stoffwechsel isolierter Organe. Du Bois' Arch. f. Physiol. 1885 S. 519.

2) J. Ries, Zeitschr. f. Biol. Bd. 47 S. 379. 1906.

3) Skutul, Über Durchströmungsapparate. Pflüger's Arch Bd. 123 S. 249.1908.

4) Hans Winterstein, Zur Kenntnis der Narkose. Zeitschr. f. allgem. Physiol., herausgeg. von Prof. Verw orn Bd. 1 H. 1 S. 21. 1901.

5) Amedeo Herlitzka, P flüger's Arch. Bd. 138 S. 188. 1911.

6) Max Verworn, Narkose S. 32. Gustav F ischer, Jena 1912.

7) Max v. Frey, Versuche über den Stoffwechsel des Muskels. Du Bois' Arch. f, Physiol. 1885 S. 583. 
wirkte. In ähnlicher Weise vermutet Verworn ${ }^{1}$ ), dass bei kontinuierlicher Durchspülung die Kapillaren dauernd undurchgängig werden. Fleischl v. Marxow ${ }^{2}$ ) schreibt dem Rhythmus im Organismus eine ganz besondere Aufgabe für den Gasaustausch im Blute zu, die er mit folgenden Worten beschreibt: „Wird eine gashaltige Flüssigkeit von einem Stosse betroffen, so verliert sie infolge der Erschütterung den Charakter einer echten Lösung, der bisherige Verband der Molekeln wird aufgehoben, und die Gasmolekeln liegen frei zwischen denen der Flüssigkeit verteilt." Es erübrigt sich, auf Fleischl v. Marxows Ansicht hier näher einzugehen; sie hat eine ausführliche Entgegnung erhalten durch die Abhandlung von N. Zuntz ${ }^{3}$ ): "Über die Kräfte, welche den respiratorischen Gasaustausch in den Lungen und in den Geweben des Körpers vermitteln". Weiterhin glaubt G. $\mathrm{Ha} \mathrm{mel}^{4}$ ), einige Vorteile der rhythmischen Durchiströmung entdeckt zu haben. Zunächst stellt er ein besseres Durchfliessen bei rhythmischer Durchspülung fest: „In Ausnahmefällen nur bleibt der Strom unter gleichmässigem Druck während eines mehrstündigen Experimentes nahezu konstant." Ferner heisst es bei ihm: „Aus diesen Angaben geht mit Evidenz hervor, dass die rhythmisch gespeisten Gefässe bei weitem mehr Flüssigkeit durchtreten lassen als die kontinuierlich durchströmten." Weiter ist $\mathrm{Hamel}$ ebenso wie $\mathrm{Kronecker}$ u. a. der Ansicht, dass ohne den Rhythmus schneller Ödembildung auftritt: „Es entstehen Ödeme, die über $50 \%$ vom Gewichte des Präparates betragen können, selbst bei niedrigen Druckhöhen. Bei rhythmischer Durchleitung kommen hingegen, wenn überhaupt, nur ganz unbeträchtliche Ödeme vor.“

H a mel bringt die besseren Resultate, die er mit rhythmischer Durchspülung erzielt hat, in Beziehung zu einer „wertvollen Eigenschaft des Herzens", die er durch seine Versuche aufgedeckt zu haben glaubt: bisher habe man das Herz als eine rhythmisch wirkende Druckpumpe angesehen, deren Stösse durch das elastische Arteriensystem aufgesammelt und in kontinuierliche Triebkraft umgesetzt

1) Verworn, a. a. 0.

2) E. Fleischl v. Marxow, Eine bisher unerkannte Wirkung des Herzschlages. Ges. Abhandl. S. 135. Leipzig 1893.

3) N. Zuntz, Pflüger's Arch. Bd. 42 S. 408.

4) Gustav Hamel, Die Bedentung des Pulses für den Blutstrom. Zeitschr. f. Biol. Bd. 25 S. 474, und Mitt. d. naturf. Gesellsch. in Bern, Sitzung vom 17. Nov. 1888. 
würden. Hamel fährt dann fort: „Uns scheint der Vorteil der rhythmischen Impulse darin zu liegen, dass die wechselnde Bewegung die Elastizität der sehr biegsamen Arterienröhren in Takt hält." $\mathrm{Er}$ bezeichnet diese heilsame Tätigkeit des Rhythmus als „innere Massage“. Endlich schreibt $\mathrm{Hamel}$ der rhythmischen Durchströmung auch einen günstigeren Einfluss auf die Ernährung der Organe zu: „Die wechselnde Spannung und Erschlaffung der Gefässe wirkt beschleunigend auf die Bewegung der Gewebssäfte in den Gefässwänden und perivaskulären Räumen und schafft dadurch bessere Ernährungsverhältnisse."

Übrigens ist zu Hamel's Versuchen noch zu bemerken, dass die Art, wie er die Menge der bei rhythmischem Strome durchfliessenden Flüssigkeit berechnet, nicht einwandfrei ist. Er bezieht die letztere auf die Zeit, während welcher der die Rhythmik der Durchspülung erzeugende Ausflusshahn geöffnet war, und übersieht, dass auch bei geschlossenem Hahne infolge der Elastizität der Gefässwände noch weiter Flüssigkeit durchströmt. Die von ihm beobachtete relativ grössere Durchflussgeschwindigkeit ergibt sich also einfach aus der Art der Berechnung.

Tatsächlich ist $\mathrm{Hamel}$ in seiner Ansicht, dass die rhythmisch gespeisten Gefässe mehr Flüssigkeit durchtreten lassen, nicht ohne Widerspruch geblieben.

Skutul ${ }^{1}$ ) schreibt über die Arbeit von Jakobj und Sobieranski, die versuchten, die normale Lebensfähigkeit der isolierten Niere zu erhalten: "Wie aus den Versuchsprotokollen leicht ersichtlich ist, lieferte die mit dem Hämatisator unter alternierendem Blutdruck durehspülte Niere kein besseres Sekretionsprodukt als die nach der früheren Methode mit konstantem Blutdruck durchspülte Niere. Auch ist die Blutmenge, die durch eine isolierte Niere unter alternierendem Druck durchströmt, keine grössere als unter konstantem Druck."

Neuerdings hat Winterstein ${ }^{2}$ ) auf Grund allgemeiner Erwägungen den Wert der rhythmischen Durchspülung überhaupt bezweifelt. Er schreibt: ${ }_{\text {Es }}$ scheint mir sehr zweifelhaft, ob einer solchen (der rhythmischen Durchspülung), die grosse Bedeutung zu-

1) Skutul, a. a. 0 .

2) H. Winterstein, Das Überleben neugeborener Sängetiere bei künstlicher Durchspülung. Wiener mediz. Wochenschr. 1910 Nr. 39. 
kommt, die ihr vielfach zuerkannt wird. Die Rhythmizität des Kreislaufes im Organismus dürfte weniger eine angestrebte als eine unvermeidbare Einrichtung sein, deren Nachteile durch die Elastizität der Gefässwände zu vermindern gesucht werden. Tatsächlich ist ja gerade dort, wo der Stoffaustausch mit den Geweben erfolgt, die Blutströmung eine völlig kontinuierliche."

Die im vorstehenden wiedergegebenen Zitate zeigen, dass zwar die Mehrzahl der Autoren sich für die rhythmische Durchspülung ausspricht, dass aber eine exakte Grundlage für diese Auffassung fehlt, da systematische Versuche über die Wirkung der rhythmischen und der kontinuierlichen Durchspülung unter genau vergleichbaren Versuchsbedingungen bisher überhaupt nicht vorliegen. Unter diesem Gesichtspunkte sind die im folgenden mitgeteilten Versuche angestellt.

\section{Methodik.}

Als Versuchsobjekt dienten Wasserfrösche. Als Durchspülungsflüssigkeit wurde Ringer-Lösung von folgender Zusammensetzung verwendet:

$$
\begin{aligned}
& 0,6 \% \mathrm{NaCl} \\
& 0,047 \% \mathrm{CaCl}_{2} \text { (wasserhaltig) } \\
& 0,042 \% \mathrm{KCl} \\
& 0,01 \% \mathrm{NaHCO}_{3} \text {. }
\end{aligned}
$$

Die Flüssigkeit, die Zimmertemperatur hatte, wurde vor jedem Versuch durch kräftiges Durchschütteln mit Sauerstoff gesättigt.

Als Kriterium der Wirkung der beiden Durchspülungsarten auf den Organismus wurde die Lebenstätigkeit der Nervenzentren benutzt, die als die empfindlichsten Organe hierzu besonders geeignet erschienen, und über deren Verhalten unter verschiedenen Versuchsbedingungen wir durch die Untersuchungen Verw or n's ${ }^{1}$ ) und seiner Schüler orientiert sind.

Die Reflexerregbarkeit der Frösche wurde durch Strychnin gesteigert; und zwar erhielten sie je nach der Grösse $0,25-0,35 \mathrm{ccm}$ einer $0,1 \%$ igen Strychninlösung $(0,25-0,35 \mathrm{mg})$ direkt in das Gefässsystem injiziert. Die Reizung erfolgte mittels eines Induktionsapparates durch kurze, tetanische Reize, die ein in den sekundären Stromkreis eingeschaltetes Metronom zuleitete. Die Reizstärke blieb während der Dauer eines Versuches stets die gleiche, ebenso der

1) Max Verworn, Ermüdung, Erschöpfung und Erholung der nervösen Zentren des Rückenmarkes. Arch. f. (Anat. u.) Physiol. 1900 Suppl. S. 152. 
Ort der Reizung, die Haut des Unterkiefers, an welcher die Elektroden durch Nähte fixiert wurden.

Als Kriterium für die Funktion der Nervenzentren bei den beiden Durchspülungsarten diente:

1. Erholung nach Ermüdung durch rhythmische Reizung;

2. Grad der Frholung nach vorangegangener Erstickung;

3. zur Erholung erforderliche Zeit nach vorangeg,angener Erstickung;

3. a) Kombination von 2. und 3 ;

4. zur Erholung erforderliche Zeit nach Narkose;

a) Alkoholnarkose;

b) Kohlensäurenarkose.

Ausser der Funktion der Nervenzentren wurde in einer Versuchsreihe auch die Ödembildung als Kriterium der Wirkung der beiden Durchströmungsarten verwendet. Hierbei konnte besonders ein Einblick in das Verhalten der Gefässwände bei rhythmischem und kontinuierlichem Strome gewonnen werden.

Zur Erläuterung der Durchspülungsmethode diene die beigegebene Zeichnung. Von der Sauerstoffbombe (a) führte die Schlauchleitung zuerst zum Druckreguliergefäss (b), von da zur Mariotte'schen Flasche $(c)$, die die Durchströmungsflüssigkeit enthielt; dann zur Pumpe (d) und schliesslich zu der unmittelbar am Herzen durchschnittenen Aorta des Frosches, in deren peripheren Stumpf die Kanüle eingebunden wurde. Die Pumpe ${ }^{1}$ ), die bei der rhythmischen Durchspülung die Arbeit des Herzens ersetzte, bestand aus einem u-förmig gebogenen Rohr, das zwei Ventile enthielt und unten in eine Halbkugel auslief, deren Boden von einer Gummimembran gebildet wurde. Gegen diese stiess rhythmisch ein Kolben, der an einer mit einem Elektromotor betriebenen Welle exzentrisch befestigt war. Die Tätigkeit der Pumpe war fein regulierbar sowohl hinsichtlich der Zahl der Kolbenstösse als auch der Grösse des Schlagvolumens.

Das Druckreguliergefäss bestand aus einem als Überdruckventil wirkenden, mit Wasser gefüllten, verstellbaren Zylinder, in den das Glasrohr, das mit der Sauerstoffbombe in Verbindung stand, eintauchte. Durch Heben und Senken des Zylinders liess sich der Druck genau einstellen und während des Versuches konstant erhalten. Bei rhythmischer Durchspülung tauchte das Glasrohr nur ganz wenig in den Zylinder ein.

1) Konstruiert von dem Mechaniker des Instituts, Herrn We stien. 
Vergl. Versuche über die Wirkung rhythm. und kontin. Durchspülung. 77

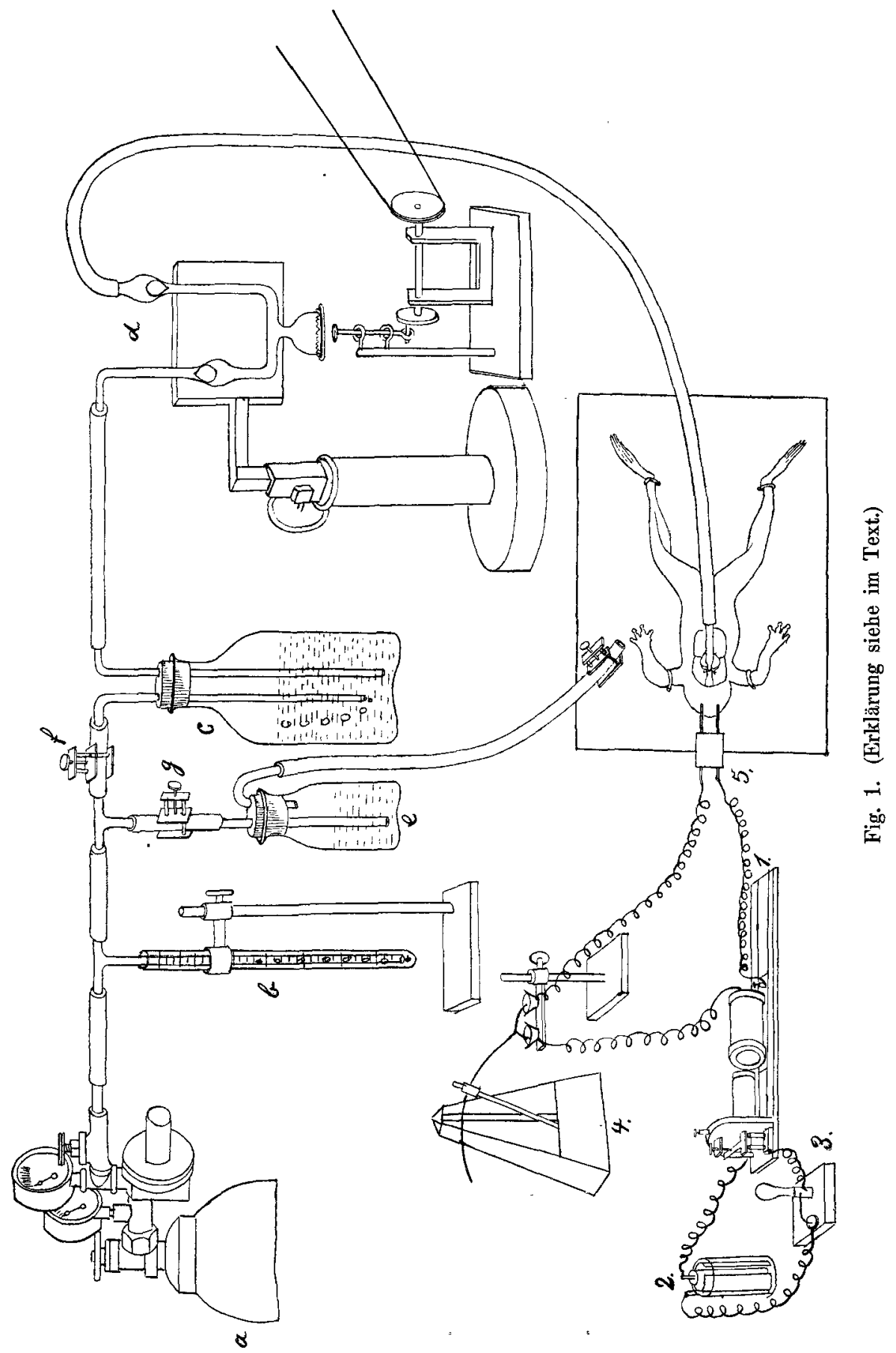


An der Mariotte'schen Flasche wurde die Menge der in das Gefässsystem des Frosehes fliessenden Flüssigkeit teils direkt von einer Skala abgelesen, teils durch die Zahl der aufsteigenden Gasblasen festgestellt. Bei sämtlichen Versuchen floss während der rhythmischen und kontinuierlichen Durchströmung in der Zeiteinheit die gleiche Flüssigkeitsmenge durch. Die von der Hauptleitung abgezweigte Nebenleitung zu der Flasche $(e)$ wurde nur bei den Versuchen mit Narkose (s. daselbst) verwendet.

Auf der Figur ist endlich noch die Reizvorrichtung dargestellt, bestehend aus Induktionsapparat (1), Element (2), Quecksilberschüssel (3), Metronom (4) und den Elektroden (5).

Kurz erwähnt sei, dass anfangs Versuche angestellt wurden, bei denen für die rhythmische und die kontinuierliche Durchspülung je ein besonderer Frosch gebraucht wurde. Bei der rhythmischen Durchströmung wurde eine Kanüle in die Vena cava inferior eingebunden, durch die die Ringer'sche Flüssigkeit dann von dem Herzen selbst rhythmisch durch das Gefässsystem gepumpt wurde. Bei der kontinuierlichen Durchströmung wurde die Kanüle in den Aortenstamm kurz vor der Teilung eingeführt und die Durchspülungsflüssigkeit von einer Mariotte'schen Flasche aus, die unter Sauerstoffdruck stand, in den Kreislauf befördert.

Es wurde immer ein Parallelversuch unter möglichst gleichen Bedingungen ausgeführt. Gleichwobl konnten infolge der zu grossen individuellen Verschiedenheiten keine vergleichbaren Resultate -gewonnen werden. Es wurde daher im folgenden stets die obige Versuchsanordnung benutzt, bei welcher die Wirkung der beiden Durchspülungsarten immer an ein und demselben Versuchstier untersucht werden konnte.

\section{Versuchsergebnisse.}

1. Erholung nach Ermüdung durch rhythmische Reizung.

Die Versuche wurden so angestellt, dass der Frosch eine bestimmte Zeit, z. B. zuerst kontinuierlich, durchspült und dann bis zur völligen Ermüdung (Reaktionslosigkeit) in der früher angegebenen Weise rhythmisch gereizt wurde. Die Zahl der Reaktionen wurde festgestellt. Dann wurde er ebenso lange rhythmisch durchströmt und hierauf wiederum gereizt und die $\mathrm{Zahl}$ der Reaktionen bestimmt. Diese diente als Maass der Erholung bei den beiden Durchströmungsarten. Die Zahl, die sich nach der ersten Durch- 
spülung ergab, kann man natürlich nicht mit in Rechnung ziehen, weil hierbei das Versuchstier vorher nicht ermüdet war. Von den so angestellten Versuchen seien folgende drei als Beispiele angeführt:

Versuch 2. 31. Januar 1912. Kleiner Frosch. 0,25 ccm Strychninlösung. Dauer der Durchspülung nach eingetretener Ermüdung je 10 Minuten.

\begin{tabular}{|c|c|}
\hline Durchspülungsart & $\begin{array}{l}\text { Zahl der Reak- } \\
\text { tionen nach } \\
\text { der Erholung }\end{array}$ \\
\hline 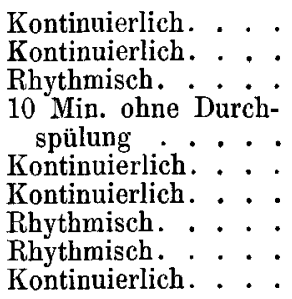 & $\begin{array}{c}107 \\
80 \\
83 \\
40 \text { (minimal) } \\
15 \\
30 \\
35 \\
30 \\
32\end{array}$ \\
\hline
\end{tabular}

Versuch 3. 1. Februar 1912. Grosser Frosch. 0,4 ccm Strychninlösung. Dauer der Durchspülung nach eingetretener Ermüdung je 10 Minuten.

\begin{tabular}{l|c}
\hline Durchspülungsart & $\begin{array}{c}\text { Zahl der Reak- } \\
\text { tionen nach } \\
\text { der Erholung }\end{array}$ \\
\hline Kontinuierlich. . . . & 80 \\
Kontinuierlich. . . . & 10 \\
Rhythmisch. . . . & 22 \\
Rhythmisch. . . . & 50 \\
Kontinuierlich. . . : & 52 \\
Kontinuierlich. . . : & 40 \\
Rhythmisch. . . . & 48 \\
Rhythmisch. . . . & 46 \\
Kontinuierlich. . . . & 47 \\
&
\end{tabular}

Versuch 4. 3. Februar 1912. Mittelgrosser Frosch. 0,35 ccm Strychninlösung. Dauer der Durchspülung nach eingetretener Ermüdung je 10 Minuten.

\begin{tabular}{l|c|c|c}
\hline Durchspülungsart & $\begin{array}{c}\text { Zahl der Reak- } \\
\text { tionen nach } \\
\text { der Erholung }\end{array}$ & Durchspülungsart & $\begin{array}{c}\text { Zahl der Reak- } \\
\text { tionen nach } \\
\text { der Erholung }\end{array}$ \\
\hline Kontinuierlich. . . . & 40 & Kontinuierlich. . . & 18 \\
Kontinuierlich. . . & 38 & Kontinuierlich . : & 17 \\
Kontinuierlich. . . & 35 & Rhythmisch. . : & 14 \\
Rhythmisch. . . . & 36 & Rhythmisch . : & 13 \\
Rhythmisch. . . . . & 30 & Kontinuierlich. . . & 13
\end{tabular}

Wenn man bei Versuch 2 von der Unregelmässigkeit absieht, die durch die starke Ermüdung nach der 10 Minuten währenden Unterbrechung der Durchspülung bedingt war, so kann man daraus entnehmen, dass die Erholung nach rhythmischer und kontinuierlicher Durchströmung gleichartig war. Dasselbe kann man von Versuch 3 sagen, der zu Anfang noch einige Schwankungen zeigte. Diese fehlten jedoch vollkommen bei Versuch 4 , in welchem infolge der immer stärker werdenden Ermüdung die Zahl der Reaktionen allmählich immer mehr abnahm, ohne irgendeinen Unterschied in der Wirkung der beiden Durchspülungsarten erkennen zu lassen. 
2. Grad der Erholung nach vorangegangener Erstickung.

Das Versuchstier wurde zunächst einige Zeit zur Entfernung des Blutes durchspiult; dann wurde die Durchspülung unterbrochen, bis Erstickungslähmung eingetreten war, zu deren Feststellung der Frosch alle 5 Minuten einmal gereizt wurde. Nach Ausbleiben der Reaktion wurde der Frosch eine bestimmte Zeit hiudurch rhythmisch bzw. kontinuierlich mit Ringer'scher Flüssigkeit durchströmt, und sodann festgestellt, wie oft er nach dieser Zeit auf die rhythmische Reizung reagierte. So wurde ein Maass des Grades der Erholung nach der vorangegangenen Erstickung bei den beiden Durchspülungsarten gewonnen. Von dieser Versuchsreihe seien die folgenden Beispiele angeführt:

Versuch 11. 15. Februar 1912. Kleiner Frosch. 0,25 ccm Strychninlösung. Dauer der Durchspülung nach eingetretener Erstickung je 10 Minuten.

\begin{tabular}{c|c|c}
\hline $\begin{array}{c}\text { Eintritt der } \\
\text { Erstickung nach }\end{array}$ & Durchspülungsart & $\begin{array}{c}\text { Zahl der Reak- } \\
\text { tionen nach } \\
\text { der Erholung }\end{array}$ \\
\hline 45 Minuten & Rhythmisch & 9 \\
32 Minuten & Kontinuierlich & 4 \\
27 Minuten & Rhythmisch & 3 \\
25 Minuten & Kontinuierlich & 1
\end{tabular}

Versuch 12. 16. Februar 1912. KJeiner Frosch. $0,25 \mathrm{ccm}$ Strychninlösung. Dauer der Durchspülung nach eingetretener Erstickung je 10 Minuten.

\begin{tabular}{c|c|c}
\hline $\begin{array}{c}\text { Eintritt der } \\
\text { Erstickung nach }\end{array}$ & Durchspülungsart & $\begin{array}{c}\text { Zabl der Reak- } \\
\text { tionen nach } \\
\text { der Erholung }\end{array}$ \\
\hline 50 Minuten & Kontinuierlich & 15 \\
37 Minuten & Rhythmisch & 3 \\
30 Minuten & Kontinuierlich & 2
\end{tabular}

Versuch 13. 19. Februar 1912. Mittelgrosser Frosch. 0,3 ccm Strychninlösung. Dauer der Durchspülung nach eingetretener Erstickung je 10 Minuten.

\begin{tabular}{c|c|c}
\hline $\begin{array}{c}\text { Eintritt der } \\
\text { Erstickung nach }\end{array}$ & Durchspülungsart & $\begin{array}{c}\text { Zahl der Reak- } \\
\text { tionen nach } \\
\text { der Erholung }\end{array}$ \\
\hline 35 Minuten & Rhythmisch & 8 \\
27 Minuten & Kontinuierlich & 3 \\
23 Minuten & Rhythmisch & 1
\end{tabular}


Vergl. Versuche über die Wirkung rhythm. und kontin. Durchspülung.

Versuch 14. 20. Februar 1912. Kleiner Frosch. 0,25 ccm Strychninlösung. Daner der Durchspülung nach eingetretener Erstickung je 10 Minuten.

\begin{tabular}{c|c|c}
\hline \hline $\begin{array}{c}\text { Eintritt der } \\
\text { Erstickung nach }\end{array}$ & Durchspülungsart & $\begin{array}{c}\text { Zahl der Reak- } \\
\text { tionen nach } \\
\text { der Erholung }\end{array}$ \\
\hline $\begin{array}{c}45 \text { Minuten } \\
40 \text { Minuten }\end{array}$ & $\begin{array}{c}\text { Kontinuierlich } \\
\text { Rhythmisch }\end{array}$ & 7 \\
\end{tabular}

Diese Versuche zeigen übereinstimmend, dass die Erholung von der Erstickung um so mangelhafter ist, je öfter der Frosch schon erstickt war, und dass ein Unterschied in dem Einfluss der rhythmischen und der kontinuierlichen Durchströmung nicht ersichtlich ist.

\section{Zur Erholung nach vorangegangener Erstickung erforderliche Zeit.}

Ganz entsprechende Resultate wurden erhalten, wenn die Zeit festgestellt wurde, nach der die Erholung eintrat. Die Versuchsmethode war im allgemeinen dieselbe wie bei den eben beschriebenen Versuchen. Nach der Erstickung wurde der Frosch 10 Minuten durchspült und hierauf alle balbe Minuten gereizt, um die Zeit zu messen, nach welcher die erste Reaktion auslösbar war.

Versuch 15. 21. Februar 1912. Kleiner Frosch. 0,25 ccm Strychninlösung.

\begin{tabular}{|c|c|c|}
\hline $\begin{array}{c}\text { Eintritt der } \\
\text { Erstickung nach }\end{array}$ & Durchspülungsart & $\begin{array}{l}\text { Erste Reaktion } \\
\text { nach }\end{array}$ \\
\hline $\begin{array}{l}45 \text { Minuten } \\
20 \text { Minuten }\end{array}$ & $\begin{array}{l}\text { Rhythmisch } \\
\text { Kontinuierlich }\end{array}$ & $\begin{array}{l}4^{1 / 2} \text { Minuten } \\
6^{1 / 2} \text { Minuten }\end{array}$ \\
\hline
\end{tabular}

Versuch 16. 22. Februar 1912. Kleiner Frosch. 0,25 ccm Strychninlösung.

\begin{tabular}{|c|c|c|}
\hline $\begin{array}{l}\text { Eintritt der } \\
\text { Erstickung nach }\end{array}$ & Durchspülungsart & $\underset{\text { nach }}{\text { Erste Reaktion }}$ \\
\hline $\begin{array}{l}45 \text { Minuten } \\
20 \text { Minuten } \\
20 \text { Minuten }\end{array}$ & $\begin{array}{l}\text { Kontinuierlich } \\
\text { Rhythmisch } \\
\text { Kontinuierlich }\end{array}$ & $\begin{array}{l}3 \text { Minuten } \\
5 \text { Minuten } \\
9 \text { Minuten }\end{array}$ \\
\hline
\end{tabular}

Versuch 17. 22. Februar 1912. Mittelgrosser Frosch. $0,3 \mathrm{ccm}$ Strychninlösung .

\begin{tabular}{|c|c|c|}
\hline $\begin{array}{l}\text { Eintritt der } \\
\text { Erstickung nach }\end{array}$ & Durchspülungsart & $\begin{array}{l}\text { Erste Reaktion } \\
\text { nach }\end{array}$ \\
\hline $\begin{array}{l}40 \text { Minuten } \\
20 \text { Minuten } \\
20 \text { Minuten } \\
20 \text { Minuten }\end{array}$ & $\begin{array}{l}\text { Rhythmisch } \\
\text { Kontinuierlich } \\
\text { Rhythmisch } \\
\text { Kontinuierlich }\end{array}$ & 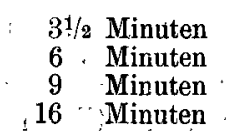 \\
\hline
\end{tabular}


Aus diesen Versuchen geht eindeutig hervor, dass die Zeit, die das Versuchstier zur Erholung von der Erstickung benötigte, mit der Wiederholung der letzteren ständig zunahm, ohne dass sich eine Differenz in der Wirkung der beiden Durchströmungsarten feststellen liess.

3a. Kombination von 2. und 3 .

Ferner wurden noch Versuche ausgeführt, die eine Kombination der beiden zuletzt mitgeteilten Versuchsarten darstellen. Es wurde nämlich nicht nur die Zeit gemessen, die bis zur Wiederkehr der ersten Reaktion gebraucht wurde, sondern es wurden auch die Reaktionen gezählt, die nach weiterer 5 Minuten währender Dauer der betreffenden Durchspülung auslösbar waren. Als Beispiel hierfür sei der folgende Versuch erwähnt.

Versuch 9. 12. Februar 1912. Mittelgrosser Frosch. 0,3 ccm Strychninlösung.

\begin{tabular}{c|c|c|c}
\hline $\begin{array}{c}\text { Eintritt } \\
\text { der Erstickung } \\
\text { nach }\end{array}$ & Durchspülungsart & $\begin{array}{c}\text { Erste Reaktion } \\
\text { nach }\end{array}$ & $\begin{array}{c}\text { Zahl der Reaktionen } \\
\text { nach weiterer Durch- } \\
\text { spülung von 5 Min. }\end{array}$ \\
\hline 65 Minuten & Kontinuierlich & 2 Minuten & 7 \\
35 Minuten & Rhythmisch & 3 Minuten & 6 \\
30 Minuten & Kontinuierlich & 9 Minuten & 3
\end{tabular}

Dieser Versuch bestätigt die Resultate der beiden anderen Versuchsreihen. Es zeigt sich eine stete Zunahme der Erholungsdauer und eine ständige Abnahme der Reaktionszahl, offenbar ohne dass die Durchspülungsart einen Einfluss darauf ausübt.

\section{Zur Erholung nach Narkose erforderliche Zeit.}

In ähnlicher Weise wie in den eben beschriebenen Versuchen wurden noch weitere Versuche angestellt, bei denen an die Stelle der asphyktischen Lähmung Narkose trat. Als Narkotikum wurde zuerst Ring er' sche Flüssigkeit verwendet, die $5 \%$ absoluten Alkohol enthielt, später Ringer'sche Flüssigkeit, die mit Kohlensäure (und Sauerstoff) geschüttelt war. Um die betäubende Flüssigkeit in das Gefässsystem des Versuchstieres gelangen zu lassen, wurde die Versuchsanordnung insofern geändert, dass an die Hauptleitung eine Nebenleitung (s. Fig.) angeschlossen wurde. Diese bestand aus einer Mariotte'schen Flasche (e), die Ringer'sche Flüssigkeit mit dem Narkotikum enthielt. Von dort ging die Leitung zum 
Aortenstamm des Frosches. Haupt- und Nebenleitung konnten durch Schraubklemmen $(f, g)$ geöffnet bzw. geschlossen werden.

Im utbrigen war die Methodik dieser Versuche die folgende. Das Versuchstier wurde zunächst mit Ringer'scher Flüssigkeit durchströmt, um das Blut zu entfernen, und dann mit der narkotisierenden Flüssigkeit bis zur Reaktionslosigkeit und noch eine bestimmte Zeit lang darüber hinaus, so dass eine vollkommene Betäubung erzielt wurde. Darauf wurde der Frosch rbythmisch bzw. kontinuierlich durchspült und durch alle halbe Minute erfolgende Reizung festgestellt, wie lange es dauerte, bis er wieder erregbar wurde.

\section{a) Alkoholnarkose.}

Versuch 19. 24. Februar 1912. Mittelgrosser Frosch. $0,3 \mathrm{ccm}$ Strychninlösung.

\begin{tabular}{c|c|c}
\hline $\begin{array}{c}\text { Eintritt } \\
\text { der Narkose nach }\end{array}$ & Durchspülungsart & $\begin{array}{c}\text { Erste Reaktion } \\
\text { nach }\end{array}$ \\
\hline $3^{1 / 2}$ Minuten & Rhythmisch & 5 Minuten \\
$3^{1 / 2}$ Minuten & Kontinuierlich & $4^{1 / 2}$ Minuten \\
$3^{1 / 2}$ Minuten & Rhythmisch & 4 Minuten \\
$3^{1 / 2}$ Minuten & Kontinuierlich & 4 Minuten \\
3 Minuten & Rhythmisch & 4 Minuten \\
3 Minuten & Kontinuierlich & 4 Minuten
\end{tabular}

Versuch 20. 26. Februar 1912. Mittelgrosser Frosch. 0,3 ccm Strychninlösung.

\begin{tabular}{c|c|c}
\hline $\begin{array}{c}\text { Eintritt } \\
\text { der Narkose nach }\end{array}$ & Durchspülungsart & $\begin{array}{c}\text { Erste Reaktion } \\
\text { nach }\end{array}$ \\
\hline 61/2 Minuten & Kontinuierlich & \\
$6^{1 / 2}$ Minuten & Rhythmisch & 6 Minuten \\
7 Minuten & Kontinuierlich & 6 Minuten \\
81/2 Minuten & Rhythmisch & 5 Minuten \\
7 Minuten & Kontinuierlich & $41 / 2$ Minuten \\
8 Minuten & Rhythmisch & 4 Minuten
\end{tabular}

Versuch 21. 27. Februar 1912. Mittelgrosser Frosch. 0,3 ccm Strychninlösung.

\begin{tabular}{c|c|c}
\hline $\begin{array}{c}\text { Eintritt } \\
\text { der Narkose nach }\end{array}$ & Durchspülungsart & $\begin{array}{c}\text { Erste Reaktion } \\
\text { nach }\end{array}$ \\
\hline $6^{1 / 2}$ Minuten & Rhythmisch & $\begin{array}{l}3 \text { Minuten } \\
5^{1 / 2} \text { Minuten }\end{array}$ \\
$5^{1 / 2}$ Minuten & Kontinuierlich & $3^{1 / 2}$ Minuten \\
$5^{1 / 2}$ Minuten & Rhythmisch & $3^{1 / 2}$ Minuten \\
6 Minuten & Kontinuierlich & 4 Minuten \\
8 Minuten & Rhythmisch & $3^{1 / 2}$ Minuten \\
& Kontinuierlich & $3^{1 / 2}$ Minuten
\end{tabular}




\section{b) Kohlensäurenarkose.}

Versuch 22. 28. Februar 1912. Mittelgrosser Frosch. 0,3 ccm Strychninlösung.

\begin{tabular}{c|c|c}
\hline $\begin{array}{c}\text { Eintritt } \\
\text { der Narkose nach }\end{array}$ & Durchspülungsart & $\begin{array}{c}\text { Erste Reaktion } \\
\text { nach }\end{array}$ \\
\hline 11/2 Minuten & Kontinuierlich & 2 Minuten \\
$21 / 2$ Minuten & Rhythmisch & $11 / 2$ Minuten \\
2 Minuten & Kontinuierlich & $11 / 2$ Minuten \\
2 Minuten & Rhythmisch & $1^{1 / 2}$ Minuten
\end{tabular}

Versuch 23. 28. Februar 1912. Grosser Frosch. $0,35 \mathrm{~cm}$ Strychninlösung.

\begin{tabular}{c|c|c}
\hline $\begin{array}{c}\text { Eintritt } \\
\text { der Narkose nach }\end{array}$ & Durchspülungsart & $\begin{array}{c}\text { Erste Reaktion } \\
\text { nach }\end{array}$ \\
\hline $1^{1 / 2}$ Minuten & Rhythmisch & $2^{1 / 2}$ Minuten \\
$1^{1 / 2}$ Minuten & Kontinuierlich & $4^{1 / 2}$ Minuten \\
$1^{1 / 2}$ Minuten & Rhythmisch & 4 Minuten \\
$1^{1 / 2}$ Minuten & Kontinuierlich & $4^{1 / 2}$ Minuten \\
$1^{1 / 2}$ Minuten & Rhythmisch & $4^{1 / 2}$ Minuten \\
$1^{1 / 2}$ Minuten & Kontinuierlich & 4 Minuten
\end{tabular}

Versuch 24. 29. Februar 1912. Mittelgrosser Frosch. $0,3 \mathrm{ccm}$ Strychninlösung.

\begin{tabular}{|c|c|c|}
\hline $\begin{array}{c}\text { Eintritt } \\
\text { der Narkose nkeh }\end{array}$ & Durchspülungsart & $\begin{array}{l}\text { Erste Reaktion } \\
\text { nach }\end{array}$ \\
\hline $\begin{array}{ll}11 / 2 & \text { Minuten } \\
1 & \text { Minute } \\
1 & \text { Minute } \\
1 & \text { Minute } \\
1 & \text { Minute } \\
1 & \text { Minute }\end{array}$ & $\begin{array}{l}\text { Kontinuierlich } \\
\text { Rhythmisch } \\
\text { Kontinuierlich } \\
\text { Rhythmisch } \\
\text { Kontinuierlich } \\
\text { Rhythmisch }\end{array}$ & $\begin{array}{l}\text { 4. Minuten } \\
4 \text { Minuten } \\
3^{1 / 2} \text { Minuten } \\
3^{1 / 2} \text { Minuten } \\
3^{1 / 2} \text { Minuten } \\
4^{1 / 2} \text { Minuten }\end{array}$ \\
\hline
\end{tabular}

Ein Überblick über die mit den beiden zuletzt mitgeteilten Versuchsmethoden gewonnenen Resultate ergibt folgendes: zwischen der kontinuierlichen und der rhythmischen Durchströmungsart liess sich kein Unterschied in dem Einfluss auf die Aufhebung der Narkose feststellen.

\section{5. Ödembildung.}

Endlich wurden noch Versuche angestellt, um die Wirkung der beiden Durchspülungsarten auf die Ödembildung zu konstatieren. Es wurde ein Frosch eine bestimmte Zeitlang rhythmisch bzw. kontinuierlich durchströmt und festgestellt, wie stark die Gewichtszunahme war, woraus man auf die Ödembildung schliessen kann. Es wurden Durchspülungen mit verschieden starker Durchflussgeschwindigkeit 
Vergl. Versuche über die Wirkung rhythm. und kontin. Durchspülung. 85

vorgenommen. Im übrigen war die Durchströmungsmethodik dieselbe wie die oben beschriebene. Es folgen jetzt die Resultate dieser Versuchsreihe.

a) Durchfiussgeschwindigkeit ca $1 \mathrm{ccm}$ pro Minute.

Versuch 1. 6. Mai 1912. Gewicht des Frosches: 71,35 g.

\begin{tabular}{l|c|c|c}
\hline \multirow{2}{*}{ Durchspülungsart } & \multirow{2}{*}{ Durchspülungsdauer } & \multicolumn{2}{|c}{ Gewichtszunahme } \\
\cline { 3 - 4 } & in Gramm & in Prozent \\
\hline Rhythmisch....... & 60 Minuten & 1,62 & 2,3 \\
Kontinuierlich. . . . . & 60 Minuten & 1,78 & 2,4
\end{tabular}

Versuch 2. 7. Mai 1912. Gewicht des Frosches: 34,60 g.

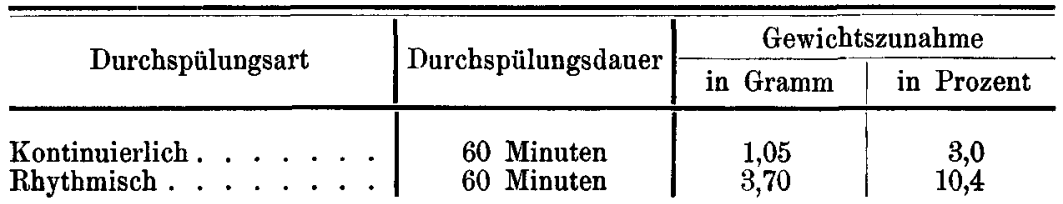

b) Durchfiussgeschwindigkeit ca 2 ccm pro Minute.

Versuch 3. 1. Mai 1912. Gewicht des Frosches: 57,35 g.

\begin{tabular}{c|c|c|c}
\hline \multirow{2}{*}{ Durchspülungsart } & \multirow{2}{*}{ Durchspülungsdauer } & \multicolumn{2}{|c}{ Gewichtszunahme } \\
\cline { 3 - 4 } & in Gramm & in Prozent \\
\hline Kontinuierlich. . . . . . & 30 Minuten & 2,10 & 3,6 \\
Rhythmisch . . . . . & 30 Minuten & 1,75 & 2,1 \\
Kontinuierlich. . . . . & 30 Minuten & 0,25 & 0,4
\end{tabular}

Versuch 4. 2. Mai 1912. Gewicht des Frosches: $90,55 \mathrm{~g}$.

\begin{tabular}{c|c|c|c}
\hline \hline \multirow{2}{*}{ Durchspülungsart } & \multirow{2}{*}{ Durchspülungsdauer } & \multicolumn{2}{|c}{ Gewichtszunahme } \\
\cline { 3 - 4 } & in Gramm & in Prozent \\
\hline Rhythmisch . . . . . . . & 30 Minuten & 0,40 & 0,4 \\
Kontinuierlich. . . . . . . & 30 Minuten & $\mathbf{1 , 5 0}$ & 1,7 \\
Rhythmisch. . . . . . & 30 Minuten & $\mathbf{2 , 8 0}$ & 3,0
\end{tabular}

c) Durchflussgeschwindigkeit ca $3 \mathrm{ccm}$ pro Minute.

Versuch 5. 3. Mai 1912. Gewicht des Frosches: 65,55 g.

\begin{tabular}{c|c|c|c}
\hline \hline \multirow{2}{*}{ Durchspülungsart } & Durchspülungsdauer & \multicolumn{2}{|c}{ Gewichtszunahme } \\
\cline { 3 - 4 } & in Gramm & in Prozent \\
\hline Rhythmisch. . . . . . . . & 30 Minuten & 8,15 & 12,5 \\
Kontinuierlich. . . . . . . & 30 Minuten & $\mathbf{5 , 4 0}$ & $\mathbf{7 , 3}$ \\
Rhythmisch . . . . . . . & 30 Minuten & $\mathbf{7 , 5 0}$ & 9,5
\end{tabular}


Paul Gerlach:

Versuch 6. 4. Mai 1912. Gewicht des Frosches: 73,25 g.

\begin{tabular}{c|c|c|c}
\hline \hline \multirow{2}{*}{ Durchspülungsart } & \multirow{2}{*}{ Durchspülungsdauer } & \multicolumn{2}{|c}{ Gewichtszunahme } \\
\cline { 4 - 4 } & & in Gramm & in Prozent \\
\hline Kontinuierlich . . . . & 30 Minuten & 1,10 & 1,5 \\
Rhythmisch . . . . & 30 Minnten & 2,55 & 3,4 \\
Kontinnierlich . . . . & 30 Minuten & 1,70 & 2,2
\end{tabular}

d) Durchflussgeschwindigkeit ca. 4 ccm pro Minute.

Versuch 7. 29. April 1912. Gewicht des Frosches: 41,75 g.

\begin{tabular}{l|c|c|c}
\hline \multirow{2}{*}{ Durchspülungsart } & Durchspülungsdauer & \multicolumn{2}{|c}{ Gewichtszunahme } \\
\cline { 3 - 4 } & in Gramm & in Prozent \\
\hline Rhythmisch . . . . & 30 Minuten & 13,20 & 31,1 \\
Kontinuierlich & 30 Minuten & 1,40 & 2,5 \\
Rhythmisch . . . . & 30 Minuten & $.7,00$ & 12,6
\end{tabular}

Bei den jetzt folgenden Versuchen wurden die beiden Durchspülungsarten an verschiedenen Fröschen angewendet.

Versuch 8. 26. April 1912. Gewicht des Frosches : 45,20 g.

\begin{tabular}{c|c|c|c}
\hline \hline Durchspülungsart & Durchspülungsdauer & \multicolumn{2}{|c}{ Gewichtszunahme } \\
\hline Kontinuierlich . . . . & 30 Minuten & 9,40 & 20,8
\end{tabular}

Versuch 9. 26. April 1912. Gewicht des Frosches: 53,35 g.

\begin{tabular}{c|c|c|c}
\hline Durchspülungsart & Durchspülungsdauer & \multicolumn{2}{|c}{ Gewichtszunahme } \\
\hline Rhythmisch Gramm & in Prozent \\
\hline . . . & 30 Minuten & 17,0 & 31,9
\end{tabular}

Versuch 10. 27. April 1912. Gewicht des Frosches: 37,00 g.

\begin{tabular}{c|c|c|c}
\hline \hline Durchspülungsart & Durchspülungsdauer & \multicolumn{2}{|c}{ Gewichtszunahme } \\
\hline Kontinuierlich . . . . & 30 Minuten & 4,90 & 13,2
\end{tabular}

Versuch 11. 27. April 1912. Gewicht des Frosches: 59,65 g.

\begin{tabular}{c|c|c|c}
\hline \hline Durchspülungsart & Durchspülungsdauer & \multicolumn{2}{|c}{ Gewichtszunahme } \\
\cline { 3 - 4 } & in Gramm & in Prozent \\
\hline Rhythmisch . . . . & 30 Minuten & 15,70 & 26,3
\end{tabular}


Ein Überblick über diese Ergebnisse zeigt, dass sich die beiden Durchströmungsarten in den Versuchsreihen a und $b$, also bei $1 \mathrm{ccm}$ bzw. 2 ecm Durchflussgeschwindigkeit pro Minute, hinsichtlich der Ödembildung als annähernd gleichwertig erweisen. Anders wird das Bild in der Versuchsreihe $\mathrm{c}$ bei $3 \mathrm{ccm}$ Durchflussgeschwindigkeit. Hier ist schon deutlich bei $\mathrm{rhyth}$ ischem Strome eine stärkere Ödembildung $\mathrm{zu}$ bemerken, was sich in noch grösserem Maasse in der Versuchsreihe $d$ bei $4 \mathrm{ccm}$ Durchflussgeschwindigkeit zeigt. Die Ursache dieser ungünstigen Wirkung des Rhythmus hat man wohl in einer Überdehnung der Gefässwände infolge der starken Pumpenstösse zu suchen.

\section{Zusammenfassung.}

Ein Vergleich der Ergebnisse sämtlicher angestellten Versuche mit den oben angeführten Angaben der Autoren ergibt folgendes: Diese schreiben im allgemeinen der kontinuierlichen Durchspülung gegenüber der rhythmischen folgende Nachteile zu:

1. schlechtere Ernährung der Gewebe;

2. schlechteres Durchfliessen der Durchspülungsflüssigkeit;

3. stärkere Ödembildung.

Keiner dieser Nachteile konnte experimentell bestätigt werden.

Bei schlechterer Ernährung der Gewebe hätte die Leistungsfähigkeit des Organismus nach kontinuierlicher Durchströmung geringer sein müssen als nach rhythmischer, was nicht der Fall war. Denn weder der Grad der Erholung noch die zum Eintritt der Erholung nach vorangegangener Ermüdung, Erstickung oder Narkose erforderliche Zeit liess einen Unterschied in der Wirkung der beiden Durchspülungsarten erkennen.

Ebensowenig konnte bei kontinuierlichem Strome ein schlechteres Durchfliessen der Durchspülungsflüssigkeit festgestellt werden. Bei beiden Durchspulungsarten nahm während der Dauer des Versuches die Menge der durchfliessenden Flüssigkeit ab. Um diese auf derselben Höhe zu erhalten, musste der Druck bzw. die Triebkraft der Pumpe langsam verstärkt werden.

Was endlich die Ödembildung anbelangt, so konnte bei mässiger Durchflussgeschwindigkeit (1-2 ecm pro Minute, welch letzterer Wert bei den früher mitgeteilten Versuchen im allgemeinen Verwendung fand) keine Differenz zwischen den beiden Durchspülungsarten konstatiert werden. Bei schnellerer Durchströmung $(3-4 \mathrm{ccm}$ 
pro Minute) zeigte sich überrasehenderweise deutlich eine schädliche Wirkung des Rhythmus, die sich in starker Ödembildung äusserte.

Auf Grund dieser Versuchsresultate ersebeint wohl die Schlussfolgerung berechtigt, dass der Rhythmik des Kreislaufes nicht die hohe Bedeutung zukommt, die man ihr vielfach zuschreibt. Sie dürfte vielmehr einfach eine mechanische Notwendigkeit darstellen, deren Nachteilen die Elastizität der Gefässwände wieder entgegenwirkt, so dass ja tatsächlich an den Orten des Stoffaustausches die Blutströmung eine völlig gleichmässige ist.

Als praktische Konsequenz dürfte sich ergeben, dass bei Versuchen mit künstlicher Zirkulation von der Anwendung rhythmischer Durchspülung keinerlei Vorteile, sondern wegen der leichteren Ödembildung eher Nachteile zu erwarten sind, und dass daher die zur Erzielung der Rhythmik erforderlichen mehr oder weniger komplizierten Vorrichtungen zum mindesten ohne Schaden forthleiben können.

Zum Schluss sei es mir erlaubt, Herrn Professor Dr. Winterstein meinen herzlichen Dank auszusprechen für die Anregung zu der vorstehenden Arbeit und seine freundliche Anleitung bei den Versuchen. 